\title{
Short-term Electrical Energy Consumption Forecasting Using GMDH-type Neural Network
}

\author{
Tsado Jacob", Usman Abraham Usman ${ }^{1,}$, , Saka Bemdoo ${ }^{2}$, Ajagun Abimbola Susan' \\ ${ }^{1}$ Department of Electrical and Electronics Engineering, Federal University of Technology Minna, Niger State, Nigeria \\ ${ }^{2}$ Transmission Company of Nigeria, TCN Abuja, Nigeria
}

Email address:

usman.abraham@futminna.edu.ng (U. A. Usman)

\section{To cite this article:}

Tsado Jacob, Usman Abraham Usman, Saka Bemdoo, Ajagun Abimbola Susan. Short-term Electrical Energy Consumption Forecasting Using GMDH-type Neural Network. Journal of Electrical and Electronic Engineering. Vol. 3, No. 3, 2015, pp. 42-47.

doi: 10.11648/j.jeee.20150303.14

\begin{abstract}
Electric load forecasting plays an important role in the planning and operation of the power system for high productivity in any institution of learning. A short-term electrical energy forecast for Gidan Kwano campus, Federal University of Technology Minna, Nigeria was carried out using GMDH-type neural network and the result was compared to that of regression analysis. GMDH-type neural network was used to train and test weekly energy consumed in the campus from September 2010 to December 2014. The neural network was trained using quadratic neural function. Root mean square error (RMSE) and mean absolute percentage error (MAPE) were used as performance indices to test the accuracy of the forecast. The neural network model gave a root mean square error (RMSE) of 0.1189 , a mean absolute percentage error (MAPE) of 0.0922 and a correlation $(\mathrm{R})$ value of 0.8995 while the regression analysis method gave a standard error of 10968.1 and a correlation $(\mathrm{R})$ value of 0.1137 . Results obtained show the efficacy of the GMDH-type neural network model in forecasting over the regression analysis method.
\end{abstract}

Keywords: Group Method of Data Handling (GMDH), Polynomial Neural Network (PNN), Short Load Term Forecasting (STLF), Mean Absolute Percentage Error (MAPE), Root Mean Square Error (RMSE)

\section{Introduction}

Electricity is one of the vital utilities for the development and higher productivity in any academic institution. Federal University of Technology Minna like many other institutions of learning in a developing country like Nigeria is faced with inadequate electricity supply. In addition, the demand for electricity in Gidan Kwano campus of Federal University of Technology Minna has increased rapidly due to the growing population, increased infrastructure and social conditions in the university. To address the shortage of electricity in the university, large investment is required. Accurate load forecasting will ensure that these investments do not waste and at the same time help distribute electricity effectively in the university campus. It will also uncover inefficiencies in the system as well as determine where savings can be made. When load forecasting is not accurately done, it will lead to improper planning and sizing of the network.

Load forecasting can be classified into three categories based on time namely: short-term load forecasting (STLF), medium term load forecasting (MTLF) and long term load forecasting (LTLF). Short-term load forecasting covers a period of one hour to one week. It is needed for efficient planning of the day-to-day operation and maintenance of the power system [1]. STLF is used to determine the electrical energy needed to meet the expected demand [2]. Medium term load forecasting covers a period ranging from one week to one year. Medium term load forecasting is important when carrying out power system maintenance, evaluation of economic dispatch and scheduling outage. Long term load forecasting covers a period of one year upwards and it is used for planning the network, future expansion plans and capital investments. This type of forecast is often complex in nature due to political and economic factors that are involved [1]. Our focus in this paper is restricted to short-term load forecasting.

Generally, load forecasting methods can be grouped into statistical and Artificial Intelligence (AI) methods. The statistical methods include regression analysis, time series 
modelling, Box-Jenkins ARIMA, exponential smoothing and similar day approach. Artificial Intelligence methods include Artificial Neural Networks (ANN), fuzzy logic, genetic algorithm (GA) and expert systems [3, 4].

The artificial intelligence methods hold an advantage over the statistical methods in their ability to predict accurately from non-linear data. Hence, neural network was used to forecast future energy consumption of Gidan Kwano campus.

\section{Literature}

A lot of work has been done on load forecasting using different techniques over the years. Generally, load forecasting methods can be grouped into conventional and artificial intelligent methods. The conventional methods include: regression analysis, time series modelling, BoxJenkins ARIMA, exponential smoothing and similar day approach while the artificial intelligent methods include: Artificial Neural Networks (ANN), fuzzy logic, genetic algorithm (GA) and expert systems. In carrying out load forecast, some factors which affect it are also considered. Some of the factors considered for load forecasting include: historical load data, weather, time factor, economic growth and random disturbance $[5,16,17]$. In Gidan Kwano campus for example, more energy is consumed during the hot season than the cold season. This is so because when the weather is hot, more energy consuming devices like air conditioners, fridges and fans are put on while the reverse is the case during the cold season.

For this work however, artificial neural network (ANN) was used to forecast energy consumed due to itps superiority over conventional methods in forecasting accurately from nonlinear data. The energy data used is nonlinear hence the ANN was used over regression method.

The human nervous system inspired the development of Artificial Neural Networks [5]. Artificial Neural Networks (ANN) comprise of processing units (or neurons) that are linked via weighted interconnections. ANN consists of a processing unit which is known as the transfer function [6]. Figure 1 illustrates a simple neuron model of the human nervous system.

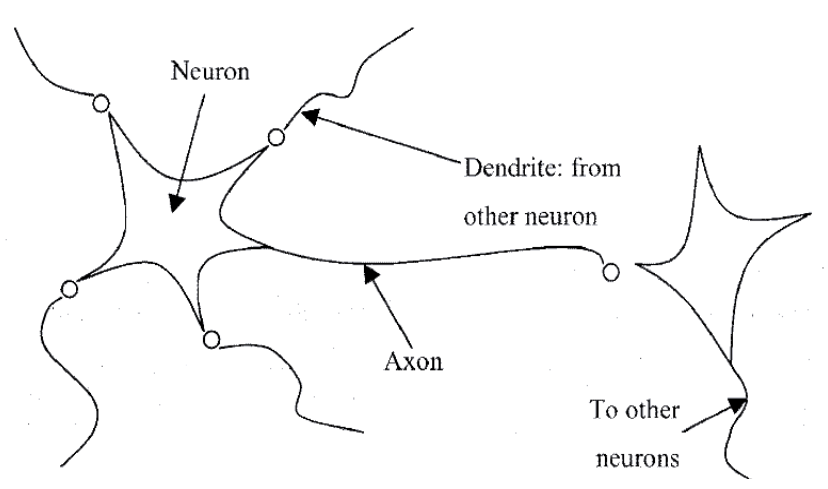

Figure 1. Neuron model of the human nervous system.

From the above model, each neuron is connected to another neuron via the dendrite or the axon. A neuron takes input via the dendrites and presents an output to the next neuron via axon. The dendrites are the interconnections [6]. This is exactly the way ANN works. For this work, GMDHtype neural network is used to predict energy consumption.

GMDH was developed by Prof. Alexey G. Ivakhnenko in 1968 at the Institute of Cybernetics in Kiev (USSR). After the initial development, thee advanced models of GMDH was developed to express multi-variable and non-linear systems [12]. The idea of GMDH is to build a model that will predict a value that is close as possible to the actual value [13]. GMDH algorithm was developed to identify non-linear relationships between inputs and outputs. GMDH algorithm can be represented as a set of neurons in which different pairs of the neurons are connected through a quadratic polynomial and therefore produce new neurons in the next layer [15]. Polynomial neural network is a flexible architecture whose structure is developed through learning. Each node of the polynomial exhibits a high degree of flexibility and realizes a polynomial mapping (linear, quadratic or cubic) between the input and the output [13]. Neural networks developed using the GMDH algorithm are called GMDH-type neural networks and are classified within the group of polynomial neural networks (PNN) [14].

The GMDH is an inductive self-organizing data driven approach. Its basic equation is called Kolmogrov-Gabor polynomial and it is expressed as shown in equation (1) below [11].

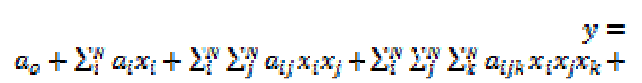

Where $\mathrm{x}_{\mathrm{i}}(\mathrm{i}=1,2 \ldots \mathrm{N})$ and $\mathrm{y}$ represent input and output variables respectively. For example, neuron can be represented with a quadratic polynomial function as shown in equation (2),

$$
y=f\left(x_{i}, x_{j}\right)=a_{0}+a_{1} x_{i}+a_{2} x_{i}+a_{2} x_{i} x_{j}+a_{4} x_{i}^{2}+a_{5} x
$$

In principle, constructed network is the composition of neurons with the mapping function $f\left(x_{i}, x_{j}\right)$. The fixed number of neurons is selected at each layer and the output of these neurons is used on the next layer [11].

\section{Materials and Methods}

The daily energy consumption data was collected from Minna Transmission station while the monthly energy consumption data was collected from the Electrical works department of Gidan Kwano campus of Federal University of Technology, Minna. The data covers a period from $1^{\text {st }}$ September, 2010 to $4^{\text {th }}$ January, 2015. The gathered data was analysed, interpreted and assigned to the model in a simplified manner.

The daily energy consumption data was compiled using MS Excel spread sheet. The weekly energy consumption was gotten by adding the daily energy consumed for a week from 
Monday to Sunday for the duration of time the data covered. The weekly energy consumption data of Gidan Kwano campus was typed in Microsoft excel. The excel file was imported to the modeling environment in CSV/XLS/XLSX format which was then imported into the neural network tool to train and validate a neural network model to forecast energy consumption of the campus. In this paper, the input to the neural network is the daily historical energy consumption data of the campus from $1^{\text {st }}$ September 2010 to 30 th December, 2014.

GMDH shell tool have various validation strategies such as k-fold cross-validation, split into training, testing and leave-one-out cross-validation. The k-fold cross-validation splits the whole dataset into $\mathrm{k}$ parts. The model is trained $\mathrm{k}$ times using k-1 parts. The independent model performance or the residuals obtained from the all the testing $\mathrm{k}$ parts are summarized in other to compare other competing models with it. In split training and testing cross-validation strategy the whole dataset is divided into two parts namely: training and testing part. The training part is used to obtain the model coefficients and the testing part is used to associate or compare all models generated. For this paper split training and testing cross-validation strategy were used. The datasets was divided in the ratio 65:35 respectively. This means 65 percent of the data was used for training and 35 percent for testing. Also, the core algorithm used for this work is GMDH neural network and the data was trained using quadratic neural function.

For the regression analysis method, the data was typed into MS excel and the regression toolbox from data analysis was used to predict energy consumption. The result from the analysis is discussed in the next section.

\section{Results and Discussion}

Result obtained from the trained model using neural network showed a correlation $(\mathrm{R})$ of 0.8995 . Since the $R$ value is close to 1 , it shows a close relationship between the actual and the predicted value, hence a good forecast. A Root Mean Square Error (RMSE) of 0.1189 and a Mean absolute percentage error (MAPE) of 0.0922 were obtained. The low error values indicate a high degree of forecasting accuracy.

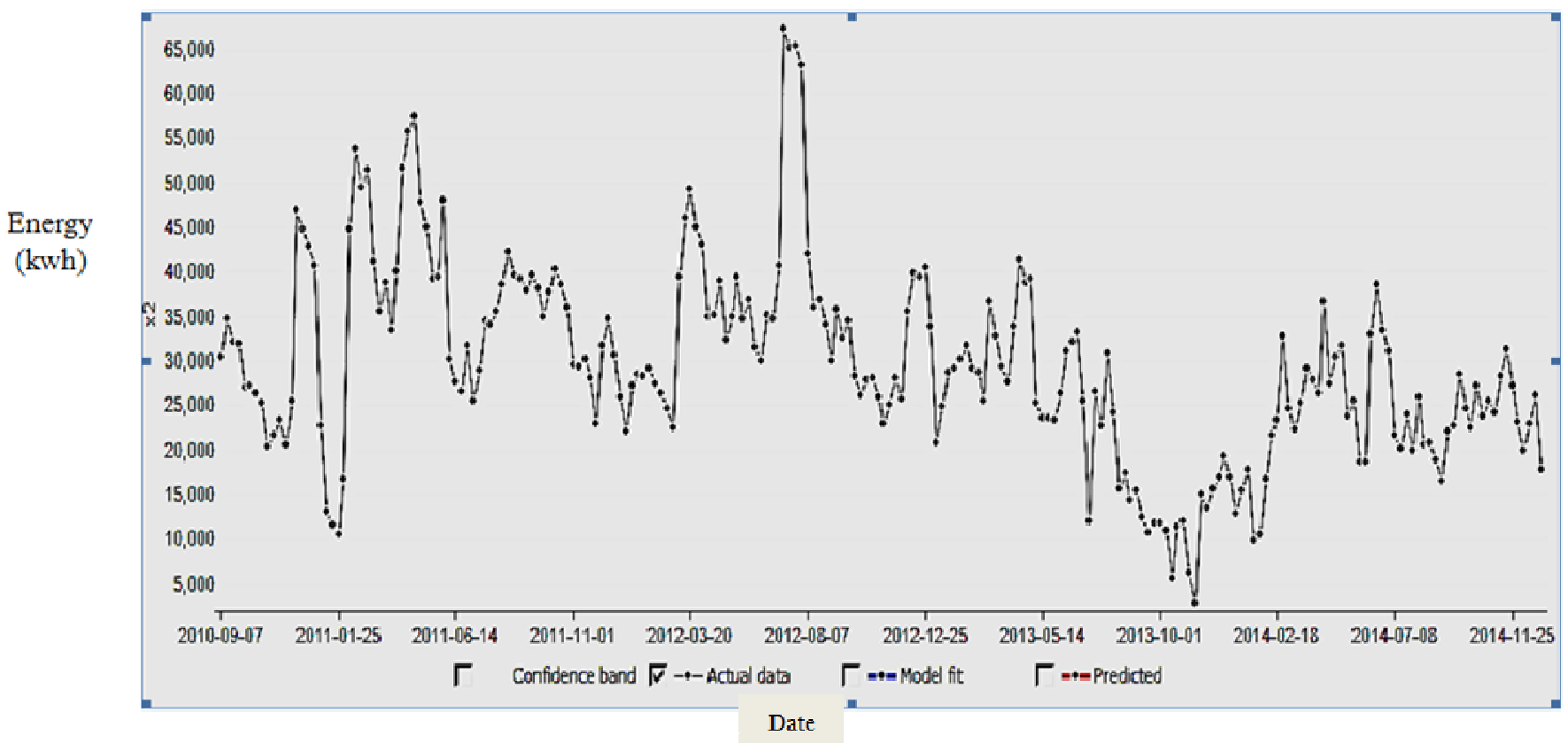

Figure 2. Actual energy consumed by Gidan Kwano campus.

The plot in figure 2 shows actual data (the historical energy consumed by Gidan Kwano campus) that was used by the neural network for training and testing. 


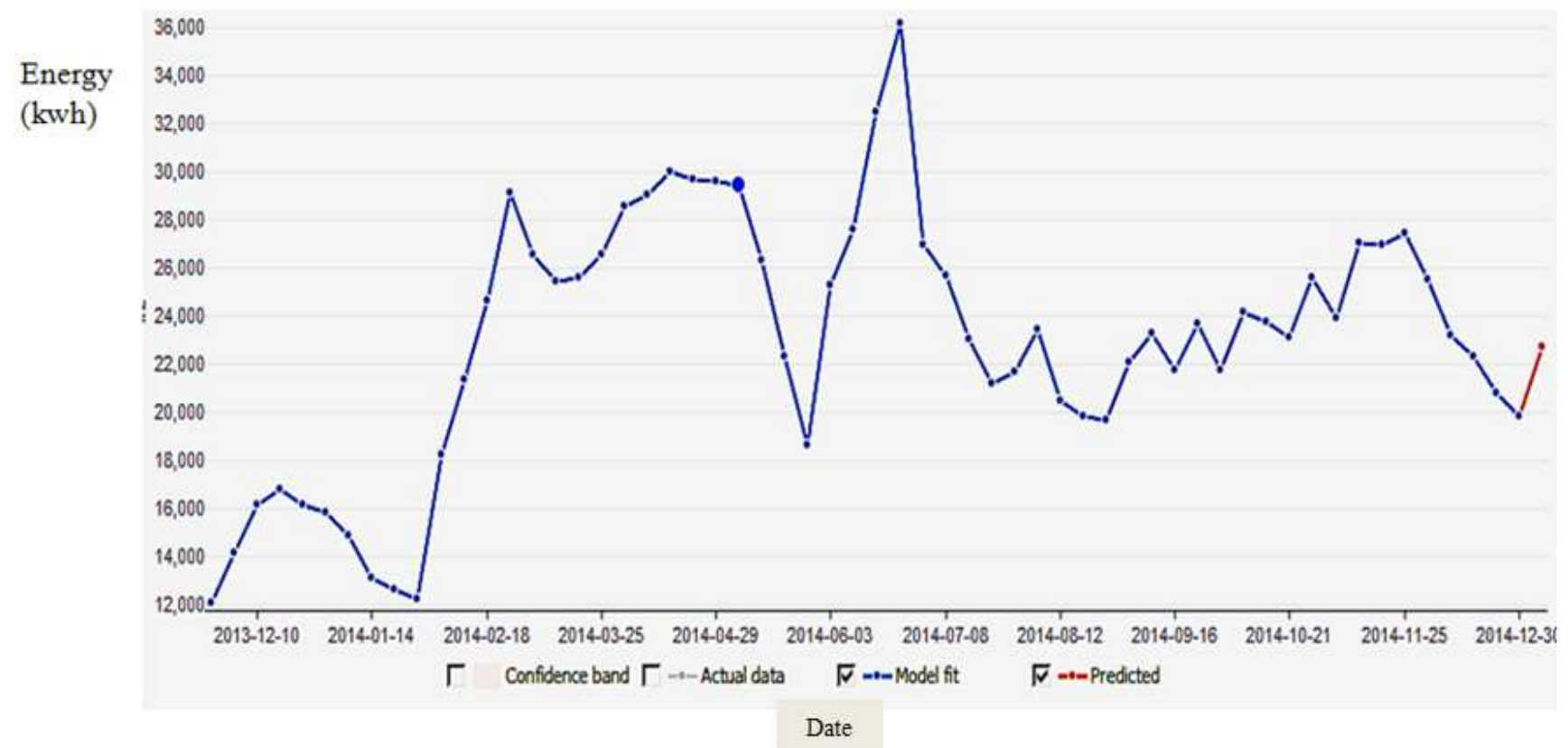

Figure 3. Model fit and predicted energy consumed.

Figure 3 show how the model values fit to the historical data. It also shows the next day prediction. The next day energy consumed from the plot is $22868 \mathrm{kwh}$

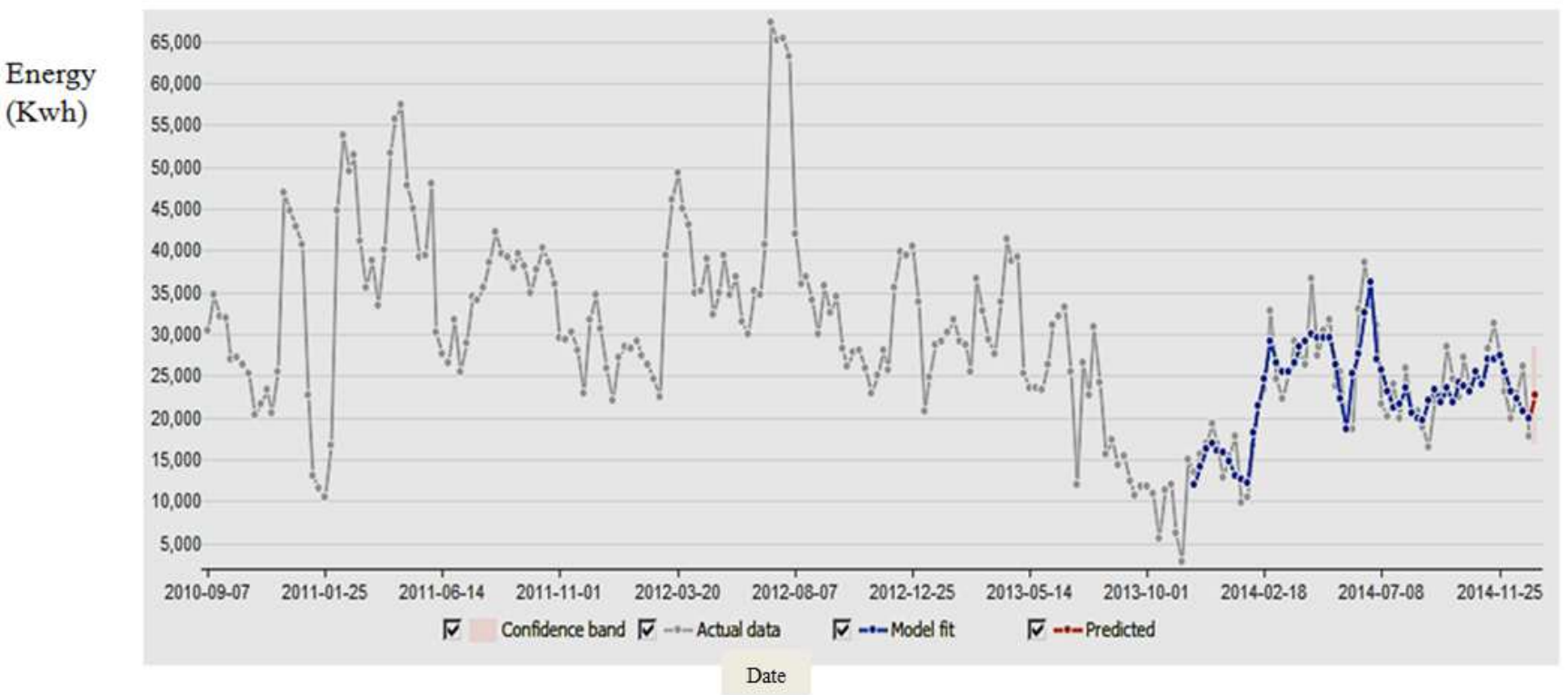

Figure 4. The actual energy consumption, the model fit and the predicted data.

Figure 4 shows the actual data, the model fit and the predicted energy consumed respectively. The actual data is the historical data that was used for training and testing and it is grey in colour. In this paper, 65 percent of the actual data was used for training while 35 percent of the actual data was used for testing. The model fit is a model value fitted to the data and is blue in colour. The predicted value is the value forecasted by the GMDH-type neural network model and is red in colour. In this paper, the next week (or step-ahead prediction) forecast is $22686 \mathrm{kwh}$.

The prediction of energy consumed at Gidan Kwano was also done using the regression analysis method. From the results, the correlation between the output and the target was found to be 0.1137 which shows a poor relationship between the actual and the predicted energy consumption. To check the forecasting accuracy, the significance $\mathrm{F}$ and the P-value was considered. A small significance of $F$ confirms the validity of the output and if this value is above 0.05 , the forecasting accuracy is poor. In this case, the value of significance $\mathrm{F}$ was found to be 0.0899 which indicates an inaccurate prediction. Also, the P-value tells whether a variable has statistically significant predictive capability in 
the presence of other variables. For a good forecasting, the Pvalue should be less than 0.05 . However, one of the values from our regression analysis was gotten to be 0.0899 which is above the 0.05 mark. This too indicates poor forecasting results. Another drawback of using regression analysis in non-linear data of Gidan Kwano is the prediction error. The standard error gotten was 10968.1 which is very high thus affecting the forecasting accuracy.

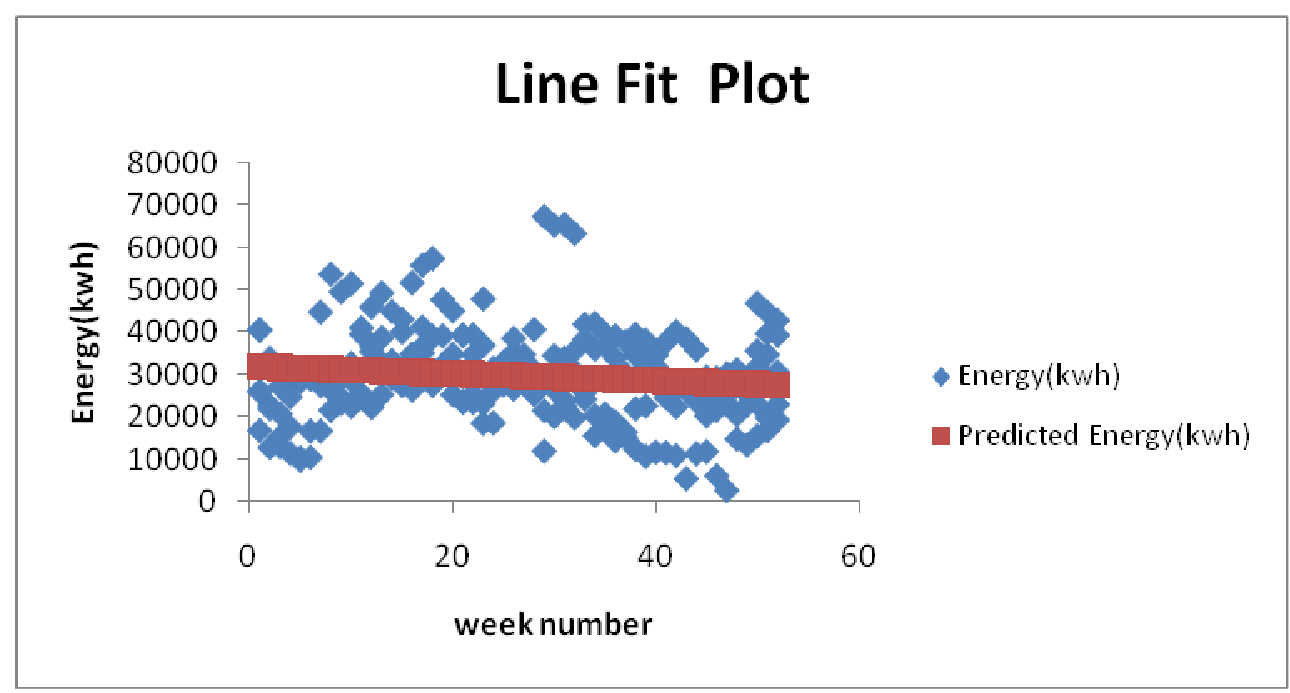

Figure 5. Line fit plot using regression method.

Figure 5 shows the plot of the actual weekly energy consumed by Gidan Kwano to the predicted energy consumed by the campus. From the plot, it can be seen that the model did not fit i.e the forecasted energy consumed is not close in value to majority of the actual energy consumed.

Table 1. Actual values of energy consumed and the predicted using the GMDH-type neural network.

\begin{tabular}{llll}
\hline S/N & DATE & $\begin{array}{l}\text { ACTUAL ENERGY } \\
\text { (Kwh) }\end{array}$ & $\begin{array}{l}\text { PREDICTED } \\
\text { ENERGY(Kwh) }\end{array}$ \\
\hline 1 & $2013-11-26$ & 13480 & 12079 \\
2 & $2013-12-03$ & 15590 & 14141 \\
3 & $2013-12-10$ & 16870 & 16193 \\
4 & $2013-12-17$ & 19360 & 16833 \\
5 & $2013-12-24$ & 16900 & 16147 \\
6 & $2013-12-31$ & 12900 & 15856 \\
7 & $2014-01-07$ & 15390 & 14850 \\
7 & $2014-01-14$ & 17780 & 13083 \\
8 & $2014-01-21$ & 9870 & 12602 \\
9 & $2014-01-28$ & 10570 & 12268 \\
10 & $2014-02-04$ & 16780 & 18262 \\
11 & $2014-02-11$ & 21640 & 21386 \\
12 & $2014-02-18$ & 23340 & 24669 \\
13 & $2014-02-25$ & 32700 & 29126 \\
14 & $2014-03-04$ & 24650 & 26570 \\
15 & $2014-03-11$ & 22330 & 25409 \\
16 & $2014-03-18$ & 25350 & 25568 \\
17 & $2014-03-25$ & 29150 & 26535 \\
18 & $2014-04-01$ & 27900 & 28513 \\
19 & $2014-04-08$ & 26410 & 29036 \\
20 & $2014-04-15$ & 36640 & 30028 \\
21 & $2014-04-22$ & 27500 & 29643 \\
22 & $2014-04-29$ & 30490 & 22330 \\
23 & $2014-05-06$ & 31720 & \\
24 & $2014-05-13$ & 23780 & 2546 \\
25 & $2014-05-20$ & 25490 & \\
\hline & & & 2960 \\
\hline
\end{tabular}

\begin{tabular}{|c|c|c|c|}
\hline $\mathbf{S} / \mathbf{N}$ & DATE & $\begin{array}{l}\text { ACTUAL ENERGY } \\
\text { (Kwh) }\end{array}$ & $\begin{array}{l}\text { PREDICTED } \\
\text { ENERGY(Kwh) }\end{array}$ \\
\hline 26 & $2014-05-27$ & 18640 & 18665 \\
\hline 27 & 2014-06-03 & 18690 & 25299 \\
\hline 28 & 2014-06-10 & 33040 & 27631 \\
\hline 29 & 2014-06-17 & 38630 & 32500 \\
\hline 30 & 2014-06-24 & 33320 & 36150 \\
\hline 31 & 2014-07-01 & 31030 & 26964 \\
\hline 32 & 2014-07-08 & 21570 & 25645 \\
\hline 33 & 2014-07-15 & 20160 & 23061 \\
\hline 34 & 2014-07-22 & 24020 & 21191 \\
\hline 35 & 2014-07-29 & 19850 & 21640 \\
\hline 36 & 2014-08-05 & 25880 & 23459 \\
\hline 37 & 2014-08-12 & 20470 & 20490 \\
\hline 38 & 2014-08-19 & 20820 & 19870 \\
\hline 39 & $2014-08-26$ & 18940 & 19704 \\
\hline 40 & 2014-09-02 & 16530 & 22065 \\
\hline 41 & 2014-09-09 & 22020 & 23259 \\
\hline 42 & 2014-09-16 & 22670 & 21764 \\
\hline 43 & $2014-09-23$ & 28460 & 23657 \\
\hline 44 & 2014-09-30 & 24680 & 21791 \\
\hline 45 & 2014-10-07 & 22450 & 24161 \\
\hline 46 & 2014-10-14 & 27130 & 23766 \\
\hline 47 & 2014-10-21 & 23780 & 23131 \\
\hline 48 & $2014-10-28$ & 25490 & 25592 \\
\hline 49 & 2014-11-04 & 24180 & 23891 \\
\hline 50 & 2014-11-11 & 28290 & 27025 \\
\hline 51 & $2014-11-18$ & 31210 & 26938 \\
\hline 52 & $2014-11-25$ & 27120 & 27406 \\
\hline 53 & 2014-12-02 & 23070 & 25506 \\
\hline 54 & 2014-12-09 & 19850 & 23199 \\
\hline 55 & $2014-12-16$ & 23020 & 22291 \\
\hline 56 & $2014-12-23$ & 26230 & 20829 \\
\hline 57 & 2014-12-30 & 17760 & 19856 \\
\hline 58 & $2015-01-06$ & & 22686 \\
\hline
\end{tabular}


Table 1 shows the actual and the predicted values. It also shows the next step prediction as $22686 \mathrm{kwh}$ (total energy consumption for the next week).

\section{Conclusion}

The energy consumed at Gidan Kwano campus of Federal University of Technology, Minna was forecasted on a shortterm basis using GMDH-type neural network and regression analysis method. The GMDH-type neural network gave better results when compared to the regression analysis method. The results are computed and the performance is measured using RMSE and MAPE. The GMDH-type neural network results show low RMSE and MAPE values while the regression analysis gave high RMSE and MAPE. These low values show a high degree of forecasting accuracy while the high values indicate a low degree of forecasting accuracy. The neural network model also gives a high correlation (R) value while the $\mathrm{R}$ value for the regression was low. This implies a close relationship between the output and the target.

From the results, the ANN performed better than the regression analysis hence, the great superiority of artificial intelligence in forecasting well from nonlinear data. The proposed STLF model can help in establishing operational plans for the University, load shedding, feeder reconfiguration and voltage control.

\section{References}

[1] A.Indira, M. Prakash, S. Pradhan, S.S. Thakur and D.V. Rajan (2014). "Short-term load forecasting of an Interconnected Grid using Neural Network". American Journal of Engineering Research (AJER), e-ISSN: 2320-0847, p-ISSN: 2320-0936, volume-03, Issue-04, pp-271-280.

[2] Seyed-Masoud Barakati, Ali Akbar Gharaveisi and Seyed Mohammad Reza Rafiei (2015). "Short-term load forecasting using mixed lazy learning method". Turkish Journal of Electrical Engineering \& Computer Sciences, Turk J Elect Eng \& Comp Sci (2015) 23: 201-211, doi: 10.3906/elk-1301134.

[3] Feinberg, E.A. and Genethliou D., (2005). Load Forecasting in: Applied Mathematics for Power Systems. State University of New York, Stony Brook, Chapter 12.

[4] Isaac, A.S., Felly- Njoku, C.F., Adewale, A.A. and Ayokunle A.A., (2014). Medium-term load forecasting of Covenant University using the Regression analysis method. Journal of Energy Technologies and Policy, ISSN 2224-3232 (paper), ISSN 2225-0573 (online), vol. 4, No. 4, 2014.

[5] Simaneka, A., (2008). Development of models for short-term load forecasting using Artificial Neural Network. Master's Thesis, Faculty of Engineering, Cape Peninsula University of Technology, November 2008.
[6] Bougaardt, G., (2002). An Investigation into the application of Artificial Neural Networks and Cluster Analysis in Long-term load Forecasting. Master's Thesis, Department of Electrical and Electronic Engineering, University of Cape Town, $1^{\text {st }}$ January, 2002.

[7] Sanjoy Das (1995). "The Polynomial Neural Network", University of Carlifonia, California 94720 1995. Information Sciences 87, 231-246 (1995), SSDI 0020-0255 (95) 00133-A.

[8] Ivan Galkin, U. Mass Lowell. "Crash Introduction to Artificial Neural Networks". Materials for UML 91.550 Data Mining Course.

[9] E. Gomez-Ramirez, K. Najim and E. Ikonen (2007). "Forecasting time series with a new architecture for polynomial artificial neural network". Applied Soft Computing 7 (2007) 1209-1216.

[10] O.A Koshulko and G.A Koshulko (2011). "Validation Strategy in combinatorial and multilayered iterative GMHD Algorithm". The $4^{\text {th }}$ International Workshop on Inductive Modelling IWIM 2011.

[11] Bon-Gil Koo, Sang-Wook Lee, Wook Kim and June Ho Park (2014). "Comparative Study of Short-term Electric Load Forecasting". 2014 Fifth International Conference on Intelligent Systems, Modeling and Simulation.

[12] Bon-Gil Koo, Heung-Seok Lee and June Ho Park (2015). "Short-term electric load forecasting based on wavelet transform and GMDH". J Electr Eng Technol. 2015; 10(?): 30-40, ISSN (Print) 1975-0102, ISSN (online) 2093-7423, http://dx.doi.org/10.5370/JEET.2015.10.2.030

[13] Huseynov A.F, Yusifbeyli N.A and Hashimov A.M (2010). "Electrical System Load forecasting with Polynomial Neural Networks (based on Combinatorial Algorithm". Modern Electric Power Systems 2010, Wroclaw, Poland, MEPS'10paper 04.3

[14] Francisco Herré́a Fernández and Fidel Hernández Lozano (2010). "GMDH Algorithm Implemented in Intelligent Identification of a Bioprocess". ABCM Symposium series in Mechatronics, vol. 4-pp 278-287.

[15] Saeed Fallahi, Meysam Shaverdi and Vahab Bashiri (2011). "Applying GMDH-type Neural Network and Genetic Algorithm for stock price prediction of Iranian cement sector", Applications and Applied Mathematics: An International Journal (AAM), vol. 6, Issue 2 (December 2011), pp 572-591, ISSN: 1932-9466

[16] Samsher, K.S. and Unde, M.G., (2012). Short-term forecasting using ANN technique. International Journal of Engineering Sciences and Engineering Technologies, Feb. 2012, ISSN: 2231-6604, volume 1, issue 2, pp: 97-107 (C) IJSEST

[17] Sanjib, M., (2008). Short-term load forecasting using computational intelligence method. Master's Thesis, Electronics and Communication Engineering (Specialization in Telematics and signal processing), National Institute of Technology, Rourkela, 2008. 